\title{
Investigación agropecuaria con enfoque agroecológico para el desarrollo de una agricultura sostenible en Nicaragua
}

\author{
Agro ecological research approach for sustainable agriculture in Nicaragua
}

\section{Pedro Enrrique Muñoz Izaguirre}

Instituto Nicaragüense de Tecnología Agropecuaria - INTA

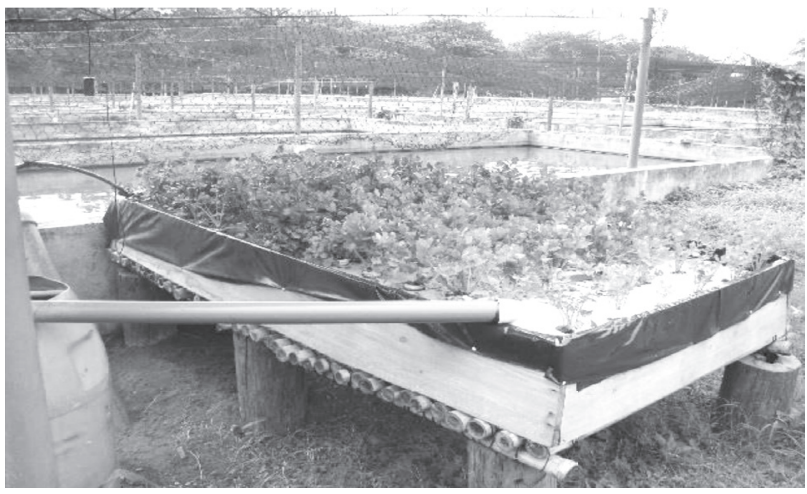

\section{RESUMEN}

El objetivo de este ensayo es aportar reflexiones acerca de la investigación agropecuaria con enfoque agroecológico como herramienta para el desarrollo de una agricultura sostenible en Nicaragua. Para brindar un panorama general de la investigación se abordan diferentes puntos de vista necesarios para su funcionamiento como son: el fortalecimiento del talento humano que realiza la actividad de investigación, la participación del productor en el proceso de investigación, la inversión público-privada y el fortalecimiento del Sistema Nicaragüense de Investigación e Innovación Agropecuaria, el que tiene la responsabilidad de plantear nuevas investigaciones para la generación de tecnologías que aporten al desarrollo de una agricultura sostenible, que tome en cuenta las interrelaciones entre los componentes físicos, biológicos y socioeconómicos, y el impacto ambiental que se suscitan dentro de los sistemas productivos. Palabras clave: sostenibilidad, agroecología, social, económico, ambiental.
The objective of this essay is to provide reflections on agricultural research with an agroecological approach as a tool for the development of sustainable agriculture in Nicaragua. In order to provide a general overview of the research, different points of view necessary for its operation are addressed, such as: the strengthening of the human talent that carries out the research activity, the participation of the producer in the research process, public-private investment and the strengthening of the Nicaraguan System of Agricultural Research and Innovation, which has the responsibility of proposing new research for the generation of technologies that contribute to the development of a sustainable agriculture that takes into account the interrelations between the physical, biological and socioeconomic components, and the environmental impact that arise within the productive systems.

Key words: Sustainability, agroecology, social, economic, environmental.

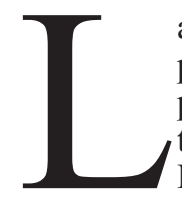

a investigación agropecuaria ha sido siempre de particular interés para la humanidad, siendo el principal factor para el incremento de la productividad agrícola en el mundo y nuestro país. El Mayor desafío que debe enfrentar la investigación agropecuaria en Nicaragua, es la generación de tecnologías sostenibles que aumenten la productividad agropecuaria, conjuntamente con la conservación de los recursos naturales, por lo que es necesario desarrollar un sistema de investigación con enfoque agroecológico con el objetivo de generar tecnologías que aporten a una agricultura sostenible, que involucre a todos los actores inmersos en la investigación agropecuaria. Sarandon y Sarandon (1993), señalan que una agricultura sostenible debe ser: suficientemente productiva, económicamente viable y financieramente posible, ecológicamente adecuada, culturalmente aceptable, socialmente justa, y técnicamente posible.

Recibido: 9 de febrero del 2017 Aceptado: 28 de abrl del 2017
El término agroecología ha llegado a significar muchas cosas, definida a groso modo, a menudo incorpora ideas sobre un enfoque de la agricultura más ligado al ambiente y más sensible socialmente, centrada no solo en la producción sino también en la sostenibilidad ecológica del sistema de producción (Altieri, 1999). Adicionalmente, la agroecología está aportando las bases científicas, metodológicas y técnicas para una nueva "revolución agraria" a escala mundial (Altieri y Toledo, 2011). Para el desarrollo de una agricultura sostenible en Nicaragua, es necesario preguntarse ¿Una agricultura sostenible, una nueva forma de investigar con enfoque agroecológico? El propósito de este trabajo es aportar reflexiones que se originan al abordar el tema de la investigación agropecuaria con enfoque agroecológico como estrategia para el desarrollo de una agricultura sostenible, enfocándose en los factores determinantes para su funcionamiento. 


\section{DESARROLLO}

A partir del planteamiento de una forma nueva de investigar, en la actualidad se habla del desafío que se presenta en la búsqueda de tecnologías orientadas en alcanzar un aumento de la productividad agropecuaria, en concordancia con la conservación de los recursos naturales, que hoy en día llamamos agricultura agroecológica.

Las instituciones de investigación en la actualidad están orientadas en el desarrollo de alternativas tecnológicas que aporten a obtener sistemas agropecuarios de bajos insumos, eficientes en el uso de la energía y principalmente diversificados. Este tipo de investigación exige un talento humano que conozcan de los componentes de los agroecosistemas y las interrelaciones que existen dentro del mismo con el objetivo de generar conocimientos que aporten a alcanzar la estabilidad a largo plazo y no el rendimiento máximo como se ha venido realizando tradicionalmente en la investigación convencional. El fortalecimiento académico del talento humano será la base del desarrollo de la investigación con enfoque agroecologico, formando capacidades en investigación con este enfoque, que sean capaces de plantear, gestionar, realizar y publicar investigaciones de alta calidad.

Un componente indispensable de la investigación con enfoque agroecológico es el agricultor, el que desempeña un papel importante en la toma de decisiones, por lo que es necesario involucrarlo en el proceso del nuevo enfoque de investigación para que pueda convencerse de la necesidad de incorporar el costo ambiental para alcanzar una producción sostenible, financieramente posible y económicamente viable.

La inversión en investigación agropecuaria es otro factor determinante para la transformación de la investigación convencional a investigación con enfoque agroecológico en Nicaragua. Stads y Beintema (2016), afirman que existen grandes brechas en el gasto y la capacidad de la investigación agropecuaria, en Latinoamérica y el Caribe han crecido considerablemente, pero aun así existen grandes diferencias entre los países, como es el caso de Nicaragua, República Dominicana, Ecuador, Panamá, Paraguay y otros países Centroamericanos, que posee un solo Instituto Nacional de investigación agropecuaria y pequeñas entidades especializadas. El país de Latinoamérica que más invierte en la región es Brasil (Stads y Beintema, 2016).

Una debilidad en investigación con enfoque agroecológico que existe en Nicaragua es la poca inversión privada, además que el presupuesto público destinado para la investigación es muy poco debido a las condiciones socioeconómicas de nuestro país, también, no existe interés del sector privado en esta actividad, ya que no destina presupuesto ni realiza investigaciones bajo su responsabilidad, lo que hacen es sumarse a las iniciativas de investigación pública para no incurrir en costos. El problema de asignaciones presupuestarias por el sector privado se centra en no valorar la generación de conocimientos que en lugar de ver el gasto en investigación agropecuaria como una inversión, la perciben como un costo adicional a sus operaciones.

En Nicaragua existe una estructura de investigación territorial que lleva por nombre Núcleo de Investigación e Innovación Territorial (NIT). El NIT es la unidad básica del Sistema Nacional de Investigación e Innovación Agropecua- ria (SNIA), que responde a las características particulares agroecológicas y productivas de los territorios, como una instancia de concertación, coordinación, planificación, implementación, seguimiento y evaluación de los procesos de investigación e innovación agropecuaria (SNIA, 2015). Participan instituciones de investigación, organizaciones de productores, así como productores y productoras individuales, organizaciones que promueven el desarrollo de la producción agropecuaria y de cadena. La coordinación del núcleo está bajo la responsabilidad del Instituto Nicaragüense de Tecnología Agropecuaria (INTA), las vice coordinaciones en una organización de productores y de investigación e innovación agropecuaria (SNIA, 2015).

Sin embargo, el avance que se tiene en el (SNIA) es organizativo en las diferentes regiones de nuestro país, se han desarrollado estructuras donde se realizan esfuerzos en conjunto con las instituciones involucradas en los diferentes NIT, llevando a cabo investigaciones interinstitucionales, pero es necesario fortalecer aún más las acciones en investigación con enfoque agroecológico, la que debe iniciar con el cambio de mentalidad de los investigadores de cada institución involucrada en esta labor, de tal forma que se cambie la manera tradicional de realizar ciencia individualista e iniciar con investigaciones desarrolladas de forma multidisciplinaria, para comprender las interrelaciones que ocurren dentro del sistema que se estudia, con el objetivo de generar conocimientos holísticos, que aporten al desarrollo de una agricultura sostenible con el uso eficiente de los recursos que posee cada sistema de producción en las diferentes zonas agroecológicas de nuestro país.

Se han generado tecnologías agropecuarias por medio de la investigación, pero de alto costo, que aportan a los componentes del sistema de producción de manera individual con enfoque reduccionista (cultivo, malezas, plaga, nutriente o suelo). Para alcanzar el desarrollo de una agricultura sostenible, el sistema Nicaragüense de Investigación e Innovación Agropecuaria tendrá que plantear una agenda de investigación agroecológica para la generación de tecnologías que abarquen el sistema en general, tomando en cuenta las interrelaciones entre los componentes físicos, biológicos, socioeconómicos y la huella ecológica o impacto ambiental que estos generan, con el propósito de alcanzar la sostenibilidad.

\section{CONCLUSIONES}

Es necesario un talento humano que conozca sobre los componentes del agro ecosistema, sus interrelaciones y funcionamiento para generar tecnologías que logren la estabilidad del sistema de producción.

Las Investigaciones con enfoque agroecológico en los diferentes núcleos de investigación territorial de nuestro país, tienen que realizarse de manera interinstitucional, multidisciplinaria y no de manera aislada como se ha venido trabajando hasta el momento.

La Investigación con enfoque agroecológico exige la participación activa del productor como sujeto de cambio en el proceso.

Es necesario fomentar la inversión público-privada en investigación con enfoque agroecológico bajo la gestión del SNIA. 


\section{REFERENCIAS BIBLIOGRÁFICAS}

Altieri, M. 1999. Bases cientificas para una agricultura sustentable. (en línea). Consultado el 13 mar. de 2017. Disponible en http:// agroeco.org/wp-content/uploads/2010/10/Libro-Agroecologia.pdf

Altieri, M; Toledo, VM. 2011. La Revolucion Agroecologica en Latinoamerica. Sociedad Cientifica Latinoamericana de Agroecologia, Berkeley, US.

Sarandon, J; Sarandon, R. 1993. Un enfoque ecologico para una agricultura sustentable. (G.F. Goñi, Ed.) Buenos Aires, AR.

SNIA (Sistema Nicaragüense de Investigación e Innovación Agropecuaria). 2015. Documento base para la instalacion del consejo regional de investigacion e innovacion agropecuaria.

Stads, Gj; Beintema, N. 2016. Investigación agropecuaria en Latinoamérica y el Caribe. Un analisis de las instituciones, la inversion y las capacidades entre paises. 